\title{
Differential Expression of rolC Results in Unique Plant Phenotypes
}

\author{
Nicole Gardner, Tracy Melberg, Manju George, and Alan G. Smith ${ }^{1}$ \\ Department of Horticultural Science, University of Minnesota, 356 Alderman Hall, St. Paul, MN 55108
}

AdDITIONAL INDEX WORDS. Nicotiana tabacum, plant architecture, leaf size, internode length

\begin{abstract}
Expression of the rolC gene in plants has been shown to cause pleiotropic effects, including decreased height. The effects of differential rolC gene expression on plant height, leaf color, root growth, leaf size, corolla length, and stem diameter were determined. Differential expression of rolC in Nicotiana tabacum L. 'Samsun' plants was achieved using the $35 \mathrm{~S}$ promoter, the light-inducible $\mathrm{rbcS}$ promoter, or the native rolC promoter. Sixteen plants from the $\mathrm{T} 1$ generation-six with the $35 \mathrm{~S}$ promoter, six with the $r b c S$ promoter, and four with the native rolC promoter - and nontransformed controls were measured for height, internode length, branch number, bud size, corolla length and diameter, root growth, and the number of days to flowering. Steady state mRNA levels of rolC were measured in roots, stems, and leaves to assess relationships between rolC expression level in specific tissues and phenotypes. Plants expressing rolC showed a wide range of phenotypes, with the largest changes in plants expressing rolC using the $35 \mathrm{~S}$ promoter, which also had the highest rolC mRNA levels. Plants expressing rolC with the rolC or $r b c S$ promoter had significant changes for many measured traits, despite rolC mRNA levels that were not significantly different from non-transformed controls. In general, as $\mathrm{rolC}$ mRNA levels increased, so did the severity of the rolC phenotype observed. Three plants, A4, A7, and B9, had unique combinations of traits that did not follow this general trend. Transformation with rolC can be useful in ornamental crops where smaller cultivars are desired.
\end{abstract}

The objective of this study was to examine the effects of differentially expressing the rolC gene using three promoters in a single species, Nicotiana tabacum. The rolC gene is one of a number of genes discovered on the Ri plasmid of Agrobacterium rhizogenes (Riker et al.) Conn. responsible for causing "hairy root" disease (van der Salm et al., 1996). Whole plants regenerated from affected "hairy" roots were found to have morphological changes, which included reduced internodes and apical dominance (Michael and Spena, 1995). The rolC gene product may have cytokinin- $\beta$-glucosidase activity that increases cytokinin levels (Casanova et al., 2004; Estruch et al., 1991). The introduction of the $r o l C$ gene into plants without other genes from the Ri plasmid has shown that plants expressing rolC demonstrate substantial changes in leaf morphology, plant height, and flower size (Michael and Spena, 1995; van der Salm et al., 1996).

Though the choice of promoter is known to have an effect on the degree of rolC phenotype observed (Fladung et al., 1993, 1997; Kurioka et al., 1992), no previous study has compared the morphological effects of rolC expressed using the three promoters of this study in a single species. Information on the effects of differentially expressing the rolC gene using three distinct promoters would be useful for manipulation of plant architecture and creating unique phenotypes in ornamental plants.

The morphological effects of rolC expression have been studied in several plant species, including Betula pendula Roth. (Piispanen et al., 2003), Dianthus caryophyllus L. (Zuker et al., 2001), N. tabacum (Scorza et al., 1994), Petunia axillaris Britton, Sters \& Poggenb. $\mathrm{x}$ (P. axillaris $\mathrm{x}$ P. hybridai Vilm.) (Winefield et al., 1999), Populus tremula L. (Fladung et al., 1997), Pyrus communis L. (Bell et al., 1999), and Solanum tuberosum L. (Fladung, 1990).

Received for publication 7 June 2005. Accepted for publication 5 Aug. 2005. The authors thank Matthias Fladung for providing the rolC gene constructs. This research was supported in part by the Minnesota Agricultural Experiment Station. Paper No. 015210148.

1To whom reprint requests should be addressed. E-mail address: alan@cbs. umn.edu
Major effects of rolC expression remain constant from species to species, and include shortened internodes, smaller flowers, altered leaf shape, reduced male fertility, and increased branching (Handa, 1992; Kiyokawa et al., 1996; Michael and Spena, 1995; Schmülling et al., 1993; Scorza et al., 1994).

Although expression of the rolC gene resulted in similar morphological changes in each plant species, the magnitude of the rolC phenotype is variable among independent transformants carrying identical rolC gene constructs (Fladung et al., 1996; Giovannini et al., 1997). Several factors, including the site of integration, copy number, mutation, somaclonal variation, and changes in expression level are cited as possible reasons for differences among independently transformed plants (Giovannini et al., 1997; Kiyokawa et al., 1996). Most of these factors, such as the site of integration, are beyond the control of the researcher, but control can be exerted over the level of expression using specific promoters. The promoter selected to express a transgene largely affects the quantity and location of gene expression. Since no research had systematically compared the effect of these three common promoters in a single species, we tested whether differences in the level and location of rolC expression would have an effect on plant phenotype and whether unique phenotypes could be obtained using a specific promoter for rolC expression.

Three promoters - the $35 \mathrm{~S}$ promoter from cauliflower mosaic virus, the native rolC promoter, and the $r b c S$ promoter-were selected to differentially express the rolC gene in this study. The $35 \mathrm{~S}$ promoter is a strong promoter expressed throughout most plant tissues, whereas the native rolC promoter is a weaker promoter that is expressed mainly in phloem cells (Schmülling et al., 1989; Yokoyama et al., 1994), and genes under control of the $r b c S$ promoter are expressed predominately in photosynthetic tissue (Fladung et al., 1997). Studies of plants expressing rolC using different promoters report differences in the degree of rolC phenotype observed (Schmülling et al., 1988). Most of the studies using rolC to modify plant growth to date have only expressed rolC under control of the $35 \mathrm{~S}$ promoter (Kurioka et 
al., 1992; Winefield et al., 1999). Although the rolC promoter has been shown to cause significant effects on plant growth when controlling the expression of rolC (Bell et al., 1999; Kiyokawa et al., 1996; Scorza et al., 1994), other studies have found no significant difference between non-transformed plants and plants expressing rolC under either the $r b c S$ promoter or the native rolC promoter (Fladung et al., 1993, 1996; Kurioka et al., 1992). A detailed study that directly compares the effects of each of these promoters in the same species would be very useful in determining the range of phenotypes and utility of each promoter in expressing rolC to produce smaller ornamental plants with unique phenotypes.

The effects of rolC expression on plant phenotype make it a candidate for the improvement of species where characteristics such as reduced height and increased branching are desirable. Several ornamental species have already been successfully transformed with rolC, including Antirrhinum majus L. (Handa, 1992), Begonia tuberhybrida Voss (Kiyokawa et al., 1996), Datura arorea Ruiz \& Pav. and D. sanguinea Ruiz \& Pav. (Giovannini et al., 1997), D. caryophyllus (Zuker et al., 2001), Eustoma grandiflorum Griesb. (Giovannini et al., 1996), Osteospermum ecklonis Norl. (Giovannini et al., 1999), P. axillaris X (P. axillaris x P. hybridai) (Winefield et al., 1999), and P. communis (Bell et al., 1999). The phenotypic improvements observed in these studies included decreased height, greater flower number, increased branching, and a reduced time to flowering.

Results from transformations of ornamental plants are encouraging, however, the effects of rolC gene expression may not always be beneficial. Several traits commonly associated with the rolC phenotype are not desirable in ornamental plants, including smaller flowers and wrinkled leaves and petals (Kiyokawa et al., 1996; Scorza et al., 1994; van der Salm et al., 1996). Differential rolC expression using specific promoters may help produce new phenotypic combinations that have potential for use in ornamental plant improvement.

To directly compare differential rolC expression with three different promoters in a single species, N. tabacum 'Samsun' plants were transformed with one of three different constructs: rolC driven by the $35 \mathrm{~S}$ promoter, the native rolC promoter, or the $r b c S$ promoter. Transformed tobacco plants in the $\mathrm{T} 1$ generation were measured for a number of horticulturally important phenotypic traits, and rolC RNA levels were measured in the leaves, stems, and roots to test whether differences in expression pattern resulted in unique phenotypes.

\section{Materials and Methods}

GENE CONSTRUCTS AND PLANT TRANSFORMATION. The rolC gene constructions were obtained from M. Fladung (Fladung et al., 1993; Spena et al., 1987). Each of the rolC constructions was grown in Agrobacterium tumefaciens (Smith \& Towns.) Conn. strain GV3101. Plants were transformed by A. tumefaciens as described previously (Horsch et al., 1985) and transformation was confirmed by polymerase chain reaction (PCR) for the $n p t I I$ gene (Saruul et al., 2002). Sixteen transformed T0 plants for each of the three different constructs were grown in the greenhouse at a temperature of $21^{\circ} \mathrm{C}$ under a photoperiod of $14 \mathrm{~h}$ day/10 $\mathrm{h}$ night, with supplemental light from sodium metal halide lamps. Each T0 plant was evaluated for a number of morphological traits and from these 16 plants - six plants expressing rolC under the $35 \mathrm{~S}$ promoter, six plants expressing rolC under the $r b c S$ promoter, and four plants expressing rolC under the native rolC promoter-were selected to represent a range of phenotypes. Selected T0 plants were crossed back to wild type $N$. tabacum 'Samsun'. Seeds from each cross were placed on germination medium containing half-strength Murashige and Skoog medium (Murashige and Skoog, 1962), $2.2 \mathrm{~g} \cdot \mathrm{L}^{-1}$ sucrose, $8 \mathrm{~g} \cdot \mathrm{L}^{-1}$ agar and $400 \mathrm{mg} \cdot \mathrm{L}^{-1}$ kanamycin to select hemizygous T1 seedlings. Five of these T1, kanamycin-resistant seedlings from each transformed line were transferred to Metro-Mix 360 growing medium (Sun Gro Horticulture, Bellevue, Wash.) and grown in a greenhouse under the same conditions as the T0 plants. Non-transformed $N$. tabacum 'Samsun' was similarly grown with the exception that no kanamycin was used in the germination medium.

RNA ANALYsis. Leaf, stem, and root tissue was collected from 8-week-old seedling T1 plants growing under 10-h days under fluorescent light with average levels of photosynthetically active radiation at $57 \mu \mathrm{mol} \cdot \mathrm{m}^{-2} \cdot \mathrm{s}^{-1}$ as determined by a quantum radiometer (LI-189; LI-COR, Lincoln, Nebr.). Leaves used in the analysis were $25 \%$ to $50 \%$ fully expanded. Tissue was collected after plants had received about $7 \mathrm{~h}$ of light that day and was ground under liquid nitrogen and stored at $-80^{\circ} \mathrm{C}$ until total RNA was extracted from the pooled plant tissue using an RNEasy Plant Mini Kit (Qiagen, Valencia, Calif.). RNA concentration was calculated from spectrophotometer optical density readings and $10 \mu \mathrm{g}$ were loaded onto a $1.2 \%$ denaturing formaldehyde gel. Gels were blotted to a GeneScreen Plus membrane [NEN Research Products, Boston (Smith et al., 1990)] and pre-hybridized, hybridized, and washed as described previously (Aguirre and Smith, 1993). The membranes were exposed to a phosphor screen (Eastman Kodak Co., Rochester, N.Y.) for a minimum of 3 $\mathrm{d}$ and scanned with the Storm PhosphoImager system (Molecular Dynamics, Sunnyvale, Calif.). Band intensities were quantified using ImageQuant software (Molecular Dynamics). All RNA blots were hybridized a second time with an 18S RNA probe for use in normalizing RNA loading between samples.

DETERMINATION OF COPY NUMBER. Two replications of approximately $200 \mathrm{~T} 1$ seeds were germinated on medium plus kanamycin. The number of resistant and susceptible plants was counted and subjected to a chi-square test to determine whether plants were segregating for more than a single locus of the rolC insert. The number of loci was confirmed by Southern blot analysis. Genomic DNA was extracted from leaf tissue and $50 \mu \mathrm{g}$ of DNA was digested with EcoRI or HindIII. Fragments were separated on a $0.7 \%$ agarose gel, blotted, and probed with ${ }^{32} \mathrm{P}-$ labeled rolC gene as described in Aguirre and Smith (1993).

MoRPHOLOGICAL MEASUREMENTS. Five T1 plants of each genotype and non-transformed controls were grown and measured in a greenhouse, and a second set of $\mathrm{T} 1$ and non-transformed plants were grown and measured 3 months later. Measurements were recorded for plant height (distance from soil line to top of the tallest growing point), internode length (length of the three internodes above the fourth node as counted from the base of the plant), number of branches, axillary bud size [rated on a scale from zero (no axillary bud) to two (axillary bud growth greater than $3 \mathrm{~cm}$ )], corolla length (from bottom of ovary to terminus) and diameter (at the terminus) of the first five flowers, number of nodes on the main stem (counted from the base to the apical meristem), stem diameter (at the ninth node), male fertility (microscopic inspection for the presence or absence of pollen on the anther), and the number of days to flowering (from transplant to soil until the first five flowers were at anthesis). Morphological measurements were taken from each plant when the first five flowers reached anthesis. 
For root length data, seeds of each T1 plant were placed on germination medium with kanamycin. After germination, five kanamycin-resistant seedlings were transferred to a petri plate of germination medium without selection. Root length was measured after 2 weeks of growth, and each plate was replicated four times.

Color data were taken with a Minolta CR-200 colorimeter (Minolta, Ramsey, N.J.). Measurements were taken for chroma, (a measure of the distance of a sample between gray and a pure color) and hue angle (where a color would be placed on a color wheel) (McGuire, 1992).

STATISTICAL ANALYSIS. Morphological and RNAmeasurements were replicated twice over time in two separate experiments, each of which used five plants of each genotype in a completely randomized design. Mean separation was determined by Tukey's studentized range (HSD) test $(P \leq 0.05)$ (StatSoft, Tulsa, Okla.). rolC RNA levels were log transformed before analysis.

\section{Results}

ROLC RNA LEVELS DIFFERED BY PROMOTER, PLANT, AND TISSUE. In all tissues examined, plants with the $35 \mathrm{~S}$ promoter (those with names beginning with the letter A) had the highest levels of rolC mRNA accumulation when compared with other promoter types in the same tissue (Table 1). In root samples, rolC mRNA levels were greater in plants carrying the rolC promoter (those

Table 1. Relative rolC mRNA levels ${ }^{\mathrm{z}}$ in stem, root, and leaf tissue of Nicotiana tabacum plants expressing rolC with either the $35 \mathrm{~S}, \mathrm{rbcS}$, or rolC promoter.

\begin{tabular}{lclll}
\hline Promoter & Plant name & Stem $^{\mathrm{y}}$ & Root $^{\mathrm{x}}$ & \multicolumn{1}{c}{ Leaf } \\
\hline 35S & A1 & $1.00 \mathrm{ma}^{\mathrm{v}}$ & $1.00 \mathrm{a}$ & $1.00 \mathrm{a}$ \\
& A3 & $0.62 \mathrm{ab}$ & $0.80 \mathrm{ab}$ & $0.74 \mathrm{ab}$ \\
& A4 & $0.36 \mathrm{~b}$ & $0.18 \mathrm{de}$ & $0.18 \mathrm{~cd}$ \\
& A7 & $0.04 \mathrm{def}$ & $0.11 \mathrm{ef}$ & $0.15 \mathrm{cde}$ \\
& A12 & $0.45 \mathrm{ab}$ & $0.44 \mathrm{bc}$ & $1.06 \mathrm{a}$ \\
$\mathrm{rbcS}$ & A14 & $0.52 \mathrm{ab}$ & $0.32 \mathrm{~cd}$ & $0.24 \mathrm{bc}$ \\
& B0 & $0.04 \mathrm{ef}$ & $0.02 \mathrm{gh}$ & $0.03 \mathrm{gh}$ \\
& B4 & $0.03 \mathrm{ef}$ & $0.01 \mathrm{hi}$ & $0.03 \mathrm{fgh}$ \\
& B9 & $0.07 \mathrm{cde}$ & $0.01 \mathrm{hi}$ & $0.06 \mathrm{defg}$ \\
& B10 & $0.11 \mathrm{~cd}$ & $0.01 \mathrm{hi}$ & $0.05 \mathrm{defg}$ \\
rolC & B13 & $0.13 \mathrm{c}$ & $0.00 \mathrm{j}$ & $0.03 \mathrm{gh}$ \\
& B14 & $0.04 \mathrm{def}$ & $0.01 \mathrm{ij}$ & $0.03 \mathrm{gh}$ \\
& C1 & $0.02 \mathrm{ef}$ & $0.03 \mathrm{~g}$ & $0.10 \mathrm{cdef}$ \\
& C6 & $0.04 \mathrm{ef}$ & $0.02 \mathrm{~g}$ & $0.02 \mathrm{gh}$ \\
Control & C8 & $0.07 \mathrm{cde}$ & $0.06 \mathrm{~g}$ & $0.01 \mathrm{gh}$ \\
& C9 & $0.05 \mathrm{cdef}$ & $0.02 \mathrm{~g}$ & $0.04 \mathrm{efg}$ \\
& D1 & $0.02 \mathrm{f}$ & $0.00 \mathrm{j}$ & $0.02 \mathrm{~h}$
\end{tabular}

zComparison of mRNA levels within a tissue relative to the A1 level. Values were calculated from rolC transformed and non-transformed plants in the T1 generation. Plants named with the letter A expressed rolC using the $35 \mathrm{~S}$ promoter; letter B plants expressed rolC using the $r b c S$ promoter; letter $\mathrm{C}$ plants expressed rolC using the native rolC promoter; and D1 plants were non-transformed. Each number represents an average of mRNA levels from two independent determinations.

yRelative rolC mRNA levels in stem tissue with $\mathrm{A} 1=1$.

Relative rolC mRNA levels in root tissue with $\mathrm{A} 1=1$.

welative rolC mRNA levels in leaf tissue with $\mathrm{A} 1=1$.

vCalculated values within a column followed by different letters are significantly different means at $P \leq 0.05$ using Tukey's HSD. Significant differences determined from original expression levels normalized for loading differences. with names beginning with the letter $\mathrm{C}$ ) than plants with the $r b c S$ promoter (those with names beginning with the letter B; Table 1). However, in leaf and stem tissues the rolC mRNA levels among plants with the rolC and $r b c S$ promoters were similar. Seven plants (A7, B0, B4, B14, C1, C6, and C9) had mRNA levels in stem tissue that were not significantly different from non-transformed plants; six plants (B0, B4, B13, B14, C6, and C9) had mRNA levels in leaf tissue that were not significantly different from non-transformed plants; and two plants (B13 and B14) had mRNA levels in root tissue that were not significantly different from non-transformed plants.

In general, rolC RNA levels in leaves, roots, and stems varied similarly among plants carrying the same construct (Table 1). Several plants, however, accumulated rolC mRNA differentially. In leaf samples, for example, the highest mRNA levels were found in A12, A3, and A1 plants, whereas in root tissue, A12 plants had significantly lower rolC mRNA levels than A1 plants. There were no significant differences among plants expressing rolC using the rolC promoter in stem and root tissue; however, in leaf tissue, $\mathrm{C} 1$ plants had significantly higher rolC mRNA levels than $\mathrm{C} 6$ and $\mathrm{C} 8$.

Variation was observed in rolC mRNA accumulation in plants transformed with the same construct. Plants expressing rolC using the $35 \mathrm{~S}$ promoter had the greatest variability in rolC mRNA levels, with a 25-fold difference between the highest (A1) and lowest (A7) plants in levels measured from stem tissue. The variation was not due to the presence of multiple rolC loci. Data from DNA-gel blots and segregation for kanamycin resistance showed that all plants except A12 and B10 contained a single copy of the rolC gene construct (data not shown). A12 and B10 plants contained at least two insertions in multiple loci. Although A12 had the highest level of rolC mRNA in leaves of all plants in other tissues it was not the highest. B10 plants did not accumulate the highest levels of rolC mRNA, even among the rbcS promoter lines.

ROLC CAUSES REDUCTIONS IN PLANT GROWTH. Expression of the rolC gene caused T1 plants to have significant reductions in height, internode length, corolla length, corolla diameter, leaf width, leaf length, stem diameter, and increases in branch number (Figs. 1 and 2). Other traits, such as days to flower, and number of nodes at flowering were not significantly different among any of the plants carrying the rolC gene constructs and non-transformed plants (data not shown). Although the effects of rolC consistently caused reductions in the size of many plant parts, there was variation in the magnitude of these effects both among and within each group of plants transformed with the same construct.

The most obvious morphological change observed among transformed plants was a reduction in height. Average plant heights ranged from $59 \mathrm{~cm}$ (A3) to $118 \mathrm{~cm}$ for the non-transformed plants (D1) (Fig. 2A). In general, plants expressing rolC using the $35 \mathrm{~S}$ promoter were the shortest of the transformed plants, followed by the $r b c S$ plants, with the rolC plants being the tallest.

Corolla length and diameter were also decreased by rolC expression (Fig. 2B). The absolute differences in corolla length between non-transformed plants (D1; $4.2 \mathrm{~cm}$ ) and the plant with the shortest corollas $(\mathrm{A} 3 ; 3.0 \mathrm{~cm})$ was small $(1.2 \mathrm{~cm})$, The plants with the shortest (A3) and the two plants with the longest (A4, A7) corolla lengths were expressing rolC using the $35 \mathrm{~S}$ promoter (Fig. 2B). Although reduced by $0.4 \mathrm{~cm}$ relative to non-transformed plants, both A4 and A7 were nearly $0.5 \mathrm{~cm}$ longer than all other plants carrying the $35 \mathrm{~S}$ promoter construct (Fig. 3 ) and 


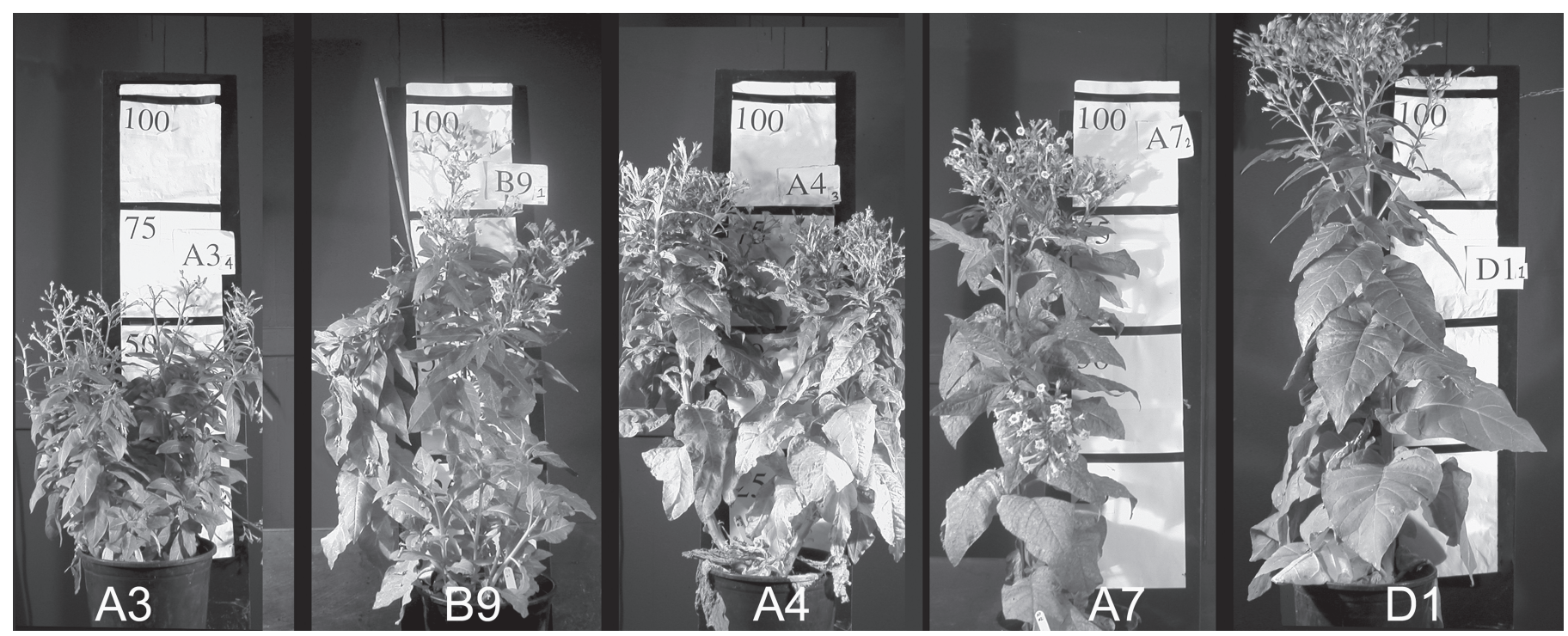

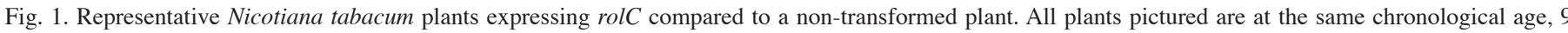

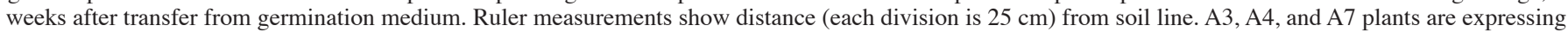
rolC using the $35 \mathrm{~S}$ promoter; plant $\mathrm{B} 9$ is expressing rolC using the $r b c S$ promoter; and plant D1 is a non-transformed control.

were similar in size to flowers from plants expressing rolC with the rolC promoter. There was no consistent association with the degree of corolla length or diameter change and a specific gene construct.

Leaf lengths of transformed plants were reduced up to $42 \%$ (A3) relative to leaves from non-transformed plants (D1), and leaf width was reduced up to $50 \%$ (A3; Fig. 2C). In general, there was a similar reduction in leaf length, leaf width, and plant height (Fig. 2 A and C). As with other traits, such as height, plants expressing rolC using the $35 \mathrm{~S}$ promoter had the largest reductions in leaf length and width; $r b c S$ plants had more intermediate reductions; and rolC plants were the most like non-transformed plants. However, the leaves of A3 plants were approximately the same size as B9 (means of leaf length and width for A3 and B9 were not significantly different), whereas the heights of these plants were significantly different (Fig. 4).

Stem diameter was reduced and followed a similar pattern of plants expressing rol $C$ using the $35 \mathrm{~S}$ promoter having the largest reductions, followed by plants using the $\mathrm{rbcS}$ and $\mathrm{rolC}$ promoter. The four plants with the smallest stem diameter (A1, A3, A12, and A14) were expressing rolC with the $35 \mathrm{~S}$ promoter and were reduced by more than one-half of a centimeter relative to nontransformed plants (D1; Fig. 2D).

Unlike most traits, which tended to be decreased with rolC expression, branch number was increased in four of the plants expressing rolC with the $35 \mathrm{~S}$ promoter. Mean branch number in the most highly branched plant (A3) was more than twice that of the non-transformed control (Fig. 2D). For most plants expressing $\mathrm{rol} C$, there was a trend toward increased branching, although most differences between plants were nonsignificant.

Plants expressing the rolC gene had leaves that were lighter and slightly more yellow in color than non-transformed plants (Fig. 2E). Seven (A1, A3, A12, A14, B4, B10, and B13) of the transformed plants showed a significant decrease in hue angle and all but two plants ( $\mathrm{C} 1$ and $\mathrm{C} 6$ ) showed a significant increase in chroma, meaning samples were more intensely colored and slightly more yellow, respectively. This change was most extreme for plants expressing rol C using the $35 \mathrm{~S}$ promoter and least extreme for plants expressing rolC using the rolC promoter in general; however, several plants did not follow this general trend. A4 plants had intermediate changes in hue and chroma in contrast to being greatly affected for other traits such as height, internode length, and stem diameter. B10 plants had greatly increased chroma and decreased hue, which was similar to plants transformed with the 35S-rolC construct, but were more intermediate in increased branch number and decreased height.

The plants expressing the rolC gene had no significant differences in root growth, despite the differences measured in other phenotypes (Fig. 2F). Observations of male fertility found no detectable pollen on the anthers of plants A1, A3, and A14.

\section{Discussion}

ROLC MRNA LEVELS WERE GREATEST IN PLANTS WITH ROLC EXPRESSED BY THE 35S PROMOTER. Differential expression of the rolC gene affected the degree of phenotypic change, but variation occurred among plants carrying each of the rolC gene constructs. Overall, the highest rolC transcript levels occurred in plants expressing rolC using the $35 \mathrm{~S}$ promoter. A direct comparison of the $35 \mathrm{~S}$ promoter to the native rolC promoter found that steady state levels of rolC mRNA were much lower with the rolC promoter (Kurioka et al., 1992).

It was expected that plants expressing rolC using the $r b c S$ promoter would have greater rolC $\mathrm{mRNA}$ levels in the leaves and stems and very low levels, if detectable, in roots, since the $r b c S$ promoter is most active in photosynthetic tissue (Fladung et al., 1997). However, most plants expressing rolC using the $r b c S$ promoter had rolC mRNA levels in leaves and stems that were low or not statistically different from non-transformed controls. This differs from studies by Fladung et al. (1993, 1997), where rolC mRNA was detected in leaves of plants with rolC expressed with the $r b c S$ promoter. The mRNA levels measured from our $r b c S$ plants may be lower because leaf mRNA was extracted from seedling leaves that were $25 \%$ to $50 \%$ fully expanded, which may differ in expression level from more mature plants and leaves (Kuhlemeier et al., 1987). Also, the seedlings were 

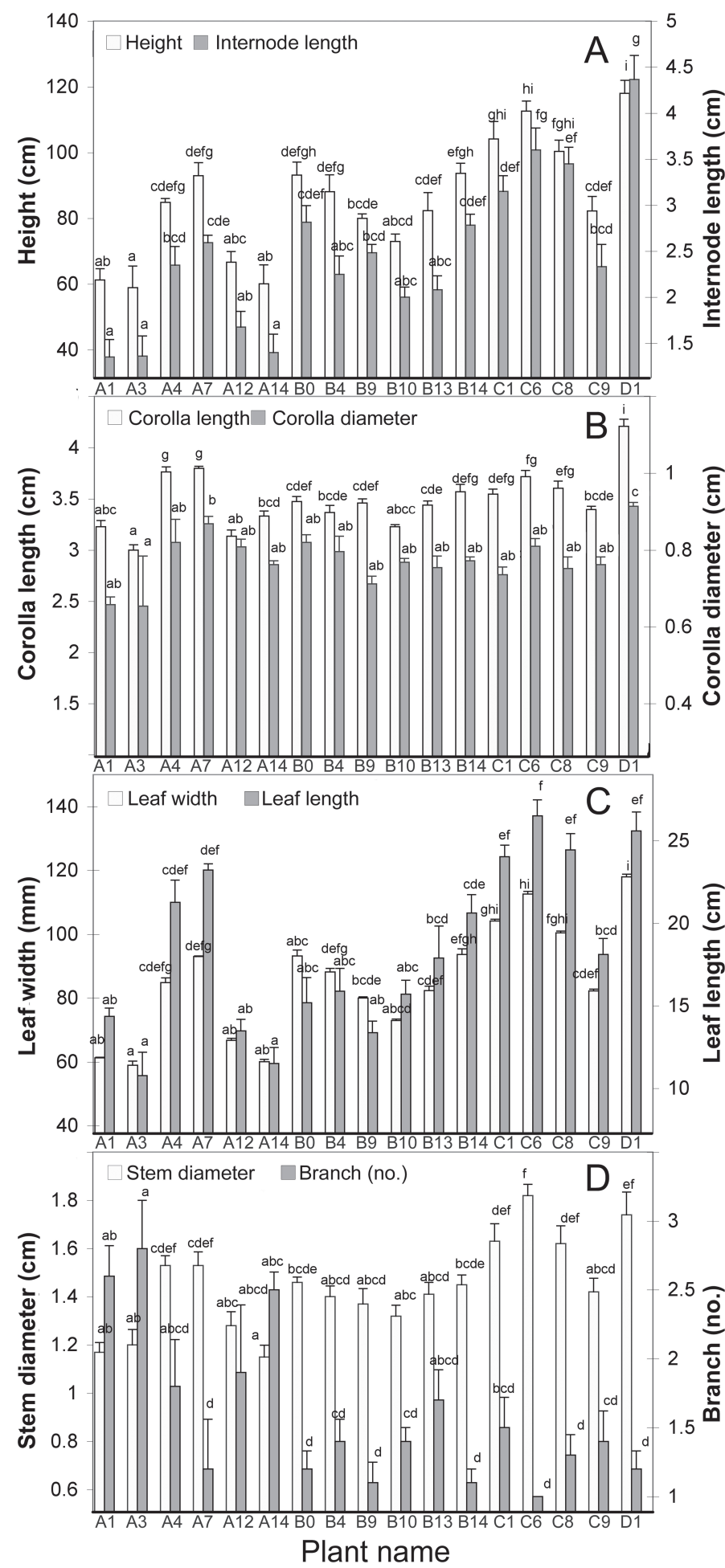

growing under lower light conditions than a greenhouse setting, which may have reduced the expression of the $r b c S$ (Kuhlemeier et al., 1987). However, the phenotypic results indicate that the rolC product is being produced in these plants.

Plants expressing the $\mathrm{rolC}$ gene with the $\mathrm{rbcS}$ and $\mathrm{rolC}$ promoters had significant morphological changes despite low levels of rolC mRNA accumulation. For example, plant $\mathrm{C} 9$ had significant changes in height and leaf length with very low levels of rolC mRNA accumulation. The possible function of the rolC gene product in alteration of growth regulators (Casanova et al., 2004;

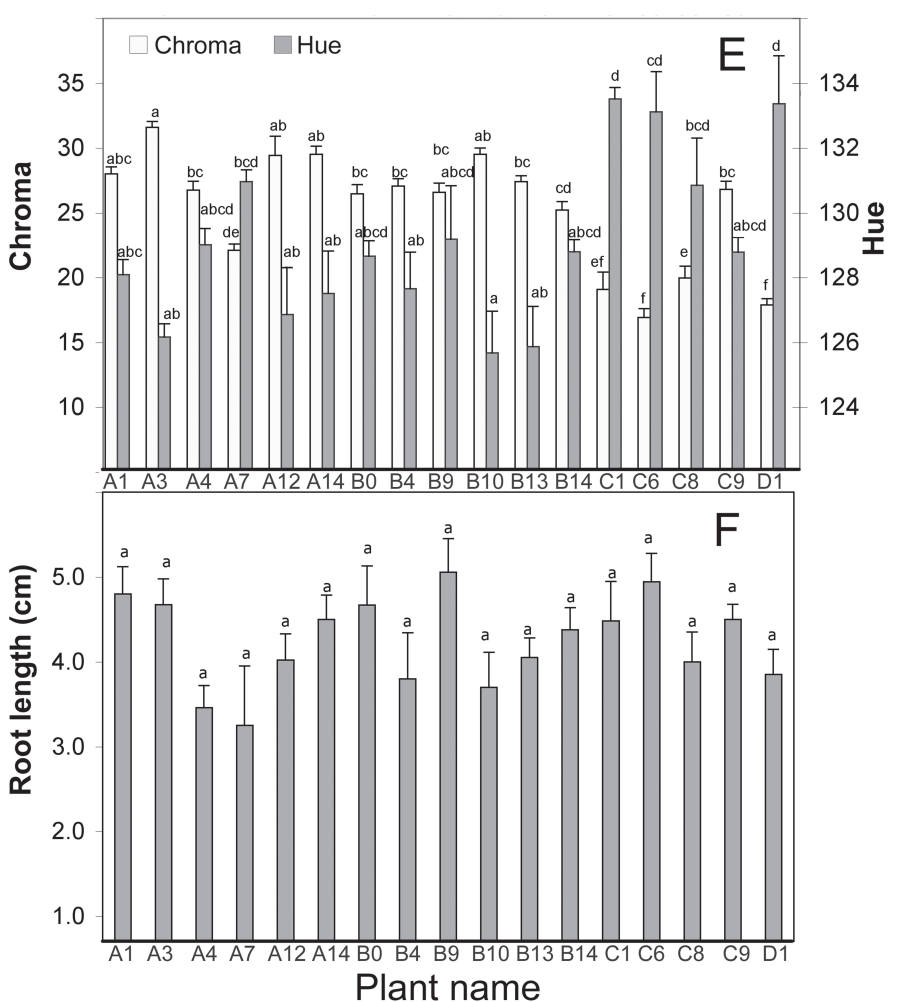

Fig. 2. (left and above) (A) Height and internode length, (B) corolla length and diameter, (C) leaf length and leaf width, (D) number of branches and stem diameter, (E) chroma and hue, and (F) root growth of Nicotiana tabacum plants expressing rolC and non-transformed controls. Plants named with the letter A expressed rolC using the $35 \mathrm{~S}$ promoter; letter B plants expressed rolC using the $\mathrm{rbcS}$ promoter; letter $\mathrm{C}$ plants expressed $\mathrm{rol} C \mathrm{C}$ using the native $\mathrm{rolC}$ promoter; and D1 plants are non-transformed controls. Each bar represents the mean of 10 different plants and the SE was used for error bars. Significant differences determined at the $P \leq 0.05$ using Tukey's HSD are indicated by different letters above each bar.

Estruch et al., 1991) could explain this apparent paradox. Small changes in growth regulator levels, distribution, or perception can have dramatic effects on growth and development (Crowell and Amasino, 1994), so if rolC does alter growth regulator levels, a small amount of rolC mRNA could still have a large effect on observed phenotype.

In general, as the rolC mRNA levels increased, so did the magnitude of the $r o l C$-induced phenotypes. The degree to which phenotypes changed, however, was not as extreme as the degree that the expression levels changed. Despite consistently higher rolC mRNA levels in A1 relative to A7, these plants were not statistically different from each other in height. Igarashi et al. (2002) also found no correlation between the rolC gene-induced phenotype and rolC $\mathrm{mRNA}$ levels in transformed 'Marubakaidou' apple rootstock (Malus prunifolia Borkh. var. ringo Asami Mo 84-A). These findings, and our own data, suggest there may be a threshold amount of rolC mRNA. Once this "threshold" amount has been reached, increased amounts of rolC mRNA would not increase the severity of phenotype observed.

MORPHOLOGICAL TRAITS ARE AFFECTED BY DIFFERENTIAL ROLC EXPRESSION. The rolC-induced phenotypes, such as decreased height, internode length, and leaf length, were common to the tobacco transformed with the three rolC gene constructs. However, there were differences among plants that could be attributed to the differential expression of the $\mathrm{rolC}$ gene using specific pro- 


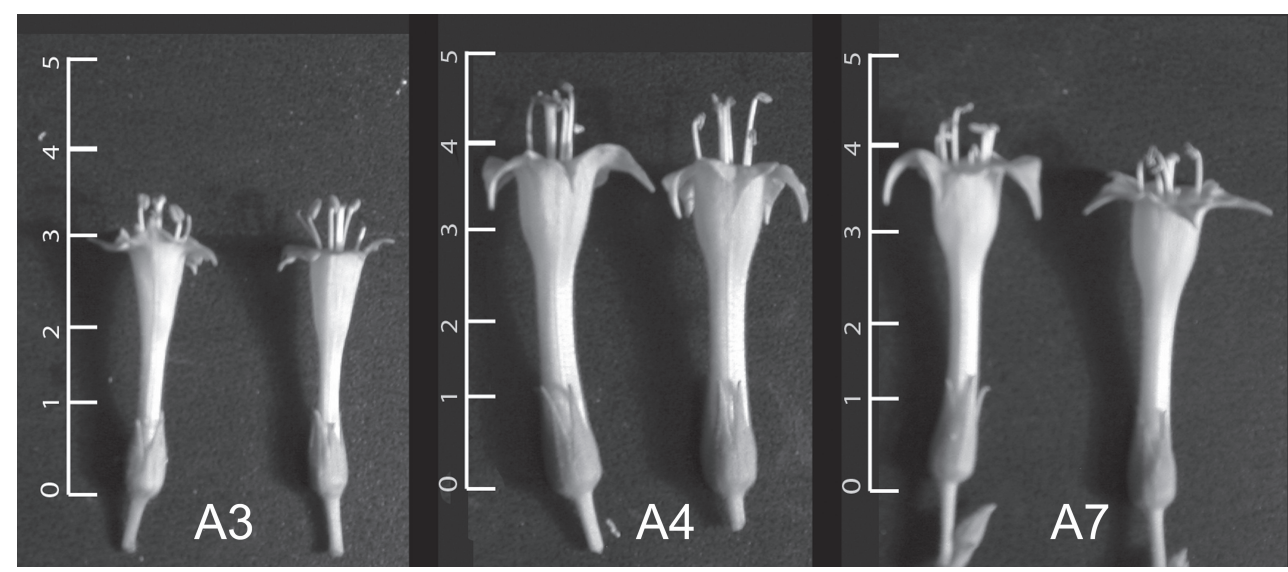

Fig. 3. Representative flowers from Nicotiana tabacum plants expressing rolC with the $35 \mathrm{~S}$ promoter (A3, A4, and A7). Ruler divisions are $1 \mathrm{~cm}$.

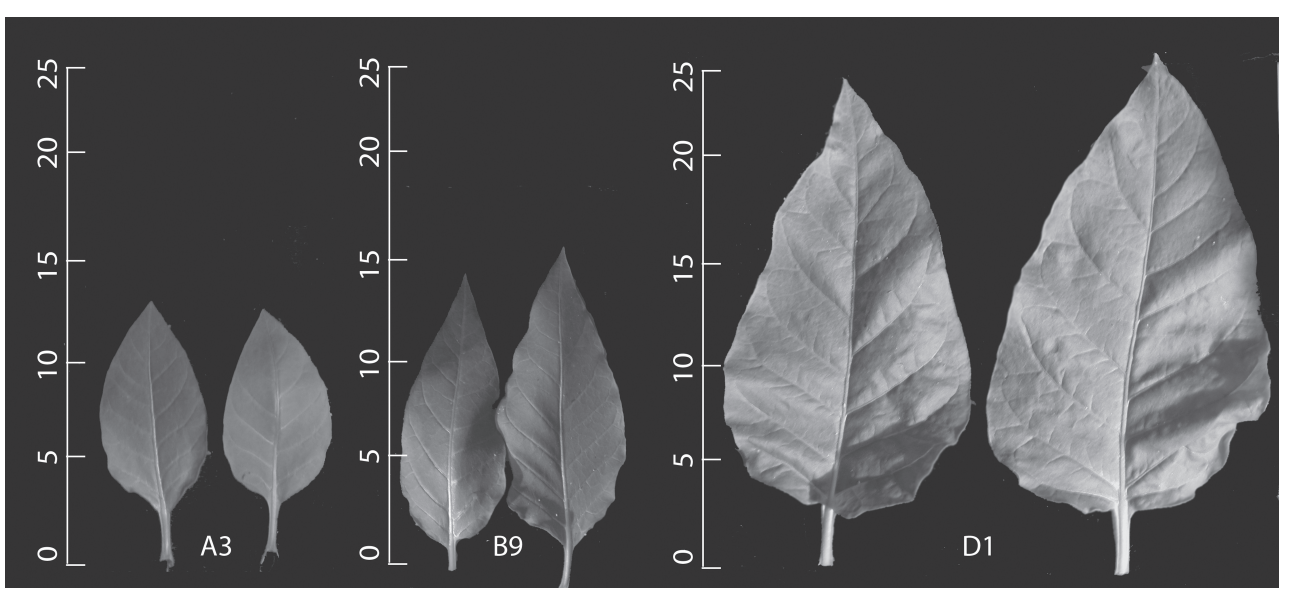

Fig. 4. Representative leaves from A3, B9, and D1 Nicotiana tabacum plants. A3 plants are expressing rolC using the $35 \mathrm{~S}$ promoter; B9 plants are expressing rolC using the $r b c S$ promoter; and D1 plants are non-transformed controls A3 and B9 leaf lengths are statistically similar, despite significant differences in the heights of the corresponding plants. Ruler divisions are shown in 5-cm increments.

moters. The $35 \mathrm{~S}$ promoter tended to produce the greatest change, followed by the $r b c S$ promoter, and the least change using the rolC promoter. The ability to manipulate phenotypic changes using specific promoters would be beneficial for ornamental plant improvement because it gives the researcher more control over a range of phenotypes.

Alterations in leaf color have been reported for plants expressing rolC (Fladung et al., 1993; Giovannini et al., 1999; Spena et al., 1989). Although the chlorophyll content of rolC plants has been measured and found to be lower (Fladung et al., 1993), this study reports the direct quantification of color changes using a Minolta CR-200 colorimeter. Chroma (the pureness of a color) and hue angle (where a color is placed on a color wheel) can be used to quantify color variations among plants. These measurements can be valuable in the selection process for ornamental plant improvement.

Root lengths, node number, and the number of days to flowering were not significantly affected by rolC expression. The lack of significant differences between transformed and non-transformed plants for root growth is an important finding for future use of rolC in ornamental plants, since plants with weaker root systems would be undesirable. The equal node number of transformed and non-transformed plants at flowering indicates that most of the height reduction in $\mathrm{rol} C$-transformed plants was due to reduced internode length. The lack of significant differences among transformed plants in the number of days to flowering differs from previous studies, which found time to flowering to be significantly reduced by the expression of rolC (Giovannini et al., 1999; Scorza et al., 1994; Winefield et al., 1999). However, two of these previous studies were on species other than $N$. tabacum, which could have been affected differently by rolC expression. Another explanation for this difference may be that in this study seeds of the T1 generation plants were used, whereas the other studies examined T0 plants that were either propagated by cuttings or grown from tissue-cultured plants. Time to flowering could be altered by differences in propagation methods.

UNIQUE PHENOTYPIC COMBINATIONS OCCUR WITH ROLC EXPRESSION. The reduction in plant height is one of the most obvious and potentially useful phenotypic effects of introducing rolC into ornamental plants. Most rolC-induced phenotypes followed the general trend of reduced height with a similar decrease in corolla length, leaf length, leaf width, corolla length, corolla width, and stem diameter. Three transformants (A4, A7, and B9), however, exhibited phenotypic combinations that did not follow this trend. These plants exemplified unique phenotypic combinations and unique rolC expression. A4 and A7 plants had the lowest levels of rolC mRNA accumulation of all plants expressing rolC with the $35 \mathrm{~S}$ promoter. However, A4 and A7 plants had corollas that were nearly $25 \%$ longer than other plants expressing rolC using the $35 \mathrm{~S}$ promoter, despite similarly reduced heights (Figs. 2A-B and 3 ). This is a desirable combination of unique phenotypes because the concomitant decrease of height and corolla length is unfavorable in ornamental plants.

In addition to displaying disproportionately large flowers for their height, A7 plants had a smaller relative change in chroma as well. The A7 plants chroma levels were only five units greater than wild type plants, whereas most other $35 \mathrm{~S}$-rolC transformants had greater changes in chroma values (Fig. 2E), which were more than 10 units increased from non-transformed controls. The decrease in leaf yellowing is in contrast to the relative decrease in height. Leaf color is important in ornamental plants, especially those valued for their foliage.

B9 plants were unique in having small leaves with less height reduction. Although mean leaf lengths for B9 and A3 plants were not statistically different, the height of B9 plants was significantly increased relative to A3 plants (Fig. $2 \mathrm{~A}$ and C). This differs from other transformants, which had a leaf size decrease that was similar to the decrease in plant height. The potential to produce 
plants with varying leaf sizes using the expression of rolC means there are greater possibilities for creating cultivars with decreased height and unique phenotypic combinations.

The expression of the rolC gene causes pleiotropic effects that have application to ornamental plant improvement. Differential expression of rolC with three different promoters produced a diverse set of rolC-induced morphological effects from similar to non-transformed plants to striking changes in height and leaf length. The variation among plants included unique combinations of $\mathrm{rolC}$-induced phenotypes. The degree of the rolC-induced phenotypes can be controlled through the choice of promoter expressing rolC, and selection of specific individuals with unique variations.

rolC expression is an effective way to produce dramatic changes in several phenotypes. For plants where methods for gene introduction are available, introduction of rolC gene constructs can be an efficient way to generate diversity in height and architecture without introducing undesirable characteristics. An increased demand for smaller-statured ornamental plants may be met through the introduction of rolC into current cultivars.

\section{Literature Cited}

Aguirre, P.J. and A.G. Smith. 1993. Molecular characterization of a gene encoding a cysteine-rich protein preferentially expressed in anthers of Lycopersicon esculentum. Plant Mol. Biol. 23:477-487.

Bell, R.L., R. Scorza, C. Srinivasan, and K. Webb. 1999. Transformation of 'Beurre Bosc' pear with the rolC gene. J. Amer. Soc. Hort. Sci. 124:570-574.

Casanova, E., A.E. Valdés, A. Zuker, B. Fernández, A. Vainstein, M.I. Trillas, and L. Moysset. 2004. rolC-transgenic carnation plants: Adventitious organogenesis and levels of endogenous auxin and cytokinins. Plant Sci. 167:551-560.

Crowell, D.N. and R.M. Amasino. 1994. Cytokinins and plant gene regulation, p. 233-241. In: D.W.S. Mok and M.C. Mok (eds.). Cytokinins: Chemistry, activity and function. CRC Press, Boca Raton, Fla.

Estruch, J.J., D. Chriqui, K. Grossman, J. Schell, and A. Spena. 1991. The plant oncogene rolC is responsible for the release of cytokinins from glucoside conjugates. EMBO J. 10:2889-2895.

Fladung, M. 1990. Transformation of diploid and tetraploid potato clones with the rolC gene of Agrobacterium rhizogenes and characterization of transgenic plants. Plant Breeding 104:295-304.

Fladung, M., A. Ballvora, and T. Schmülling. 1993. Constitutive or lightregulated expression of the rolC gene in transgenic potato plants has different effects on yield attributes and tuber carbohydrate composition. Plant Mol. Biol. 23:749-757.

Fladung, M., S. Kumar, and M.R. Ahuja. 1997. Genetic transformation of Populus gentoypes with different chimaeric gene constructs: Transformation efficiency and molecular analysis. Transgenic Res. 6:111-121.

Fladung, M., H.J. Muhs, and M.R. Ahuja. 1996. Morphological changes in transgenic Populus carrying the rolC gene from Agrobacterium rhizogenes. Silvae Genet. 45:349-354.

Giovannini,A., N. Pecchioni, and A. Allavena. 1996. Genetic transformation of lisianthus (Eustoma grandiflorum Griesb.) by Agrobacterium rhizogenes. J. Genet. Breeding 50:35-40.

Giovannini, A., N. Pecchioni, M. Rabaglio, and A. Allavena. 1997. Characterization of ornamental Datura plants transformed by Agrobacterium rhizogenes. In Vitro Cell. Dev. Biol. 33:101-106.

Giovannini, A., M. Zottini, G. Morreale, A. Spena, and A. Allavena. 1999. Ornamental traits modification by rol genes in Osteospermum ecklonis transformed with Agrobacterium tumefaciens. In Vitro Cell. Dev. Biol. Plant 35:70-75.

Handa, T. 1992. Genetic transformation of Antirrhinum majus L. and inheritance of altered phenotype induced by Ri T-DNA. Plant Sci. 81:199-206.
Horsch, R., J. Fry, N. Hoffman, M. Wallroth, D. Eichholtz, S. Rogers, and R. Fraley. 1985. A simple and general method for transferring genes into plants. Science 227:1229-1231.

Igarashi, M., H. Ogasawara, Y. Hatsuyama, A. Saito, and M. Suzuki. 2002. Introduction of rolC into Marubakaidou [Malus prunifolia Borkh. var. ringo Asami Mo 84-A] apple rootstock via Agrobacterium tumefaciens. Plant Sci. 163:463-473.

Kiyokawa, S., Y. Kikuchi, H. Kamada, and H. Harada. 1996. Genetic transformation of Begonia tuberhybida by Ri rol genes. Plant Cell Rpt. 15:606-609.

Kuhlemeier, C., P.J. Green, and N. Chua. 1987. Regulation of gene expression in higher plants. Ann. Rev. Plant Physiol. 38:221-257.

Kurioka, Y., Y. Suzuki, H. Kamada, and H. Harada. 1992. Promotion of flowering and morphological alterations in Atropa belladonna transformed with a CaMV 35S-rolC chimeric gene of the Ri plasmid. Plant Cell Rpt. 12:1-6.

McGuire, R.G. 1992. Report of objective color measurements. HortScience 27:1254-1255.

Michael, T. and A. Spena. 1995. The plant oncogenes rolA, B, and C from Agrobacterium rhizogenes, p. 207-221. In: K.M.A. Gartland and M.R. Davey (eds.). Methods in molecular biology, Vol. 44: Agrobacterium protocols. Humana Press, Totowa, N.J.

Murashige, T. and F. Skoog. 1962. Arevised medium for rapid growth and bio-assay with tobacco tissue cultures. Physiol. Plant 75:325-332.

Piispanen, R., T. Aronen, X. Chen, P. Saranpää, and H. Häggman. 2003. Silver birch (Betula pendula) plants with aux and rol genes show consistent changes in morphology, xylem structure and chemistry. Tree Physiol. 23:721-733.

Saruul, P., F. Srienc, D.A. Somers, and D.A. Samac. 2002. Production of a biodegradable plastic polymer, poly-ß-hydroxybutyrate, in transgenic alfalfa. Crop Sci. 42:919-927.

Schmülling, T., H. Röhrig, S. Pilz, R. Walden, and J. Schell. 1993. Restoration of fertility by antisense RNA in genetically engineered male sterile tobacco. Theor. Appl. Genet. 237:385-394.

Schmülling, T., J. Schell, and A. Spena. 1988. Single genes from Agrobacterium rhizogenes influence plant development. EMBO J. 7:2621-2629.

Schmülling, T., J. Schell, and A. Spena. 1989. Promoters of the rolA, rolB and rolC genes of Agrobacterium rhizogenes are differently regulated in transgenic plants. Plant Cell 1:665-670.

Scorza, R., T.W. Zimmerman, J.M. Cordts, K.J. Footen, and M. Ravelonandro. 1994. Horticultural characteristics of transgenic tobacco expressing the rolC gene from Agrobacterium rhizogenes. J. Amer. Soc. Hort. Sci. 119:1091-1098.

Smith, A.G., C.S. Gasser, K.A. Budelier, and R.T. Fraley. 1990. Identification and characterization of stamen and tapetal specific genes from tomato. Mol. Gen. Genet. 222:9-16.

Spena,A., R.B.Aalen, and S.C. Schuzle. 1989. Cell-autonomous behavior of the rolC gene of Agrobacterium rhizogenes during leaf development: A visual assay for transposon excision in transgenic plants. Plant Cell $1: 1157-1164$.

Spena, A., T. Schmülling, C. Koncz, and J.S. Schell. 1987. Independent and synergistic activity of rolA, $B$ and $C$ loci in stimulating abnormal growth in plants. EBMO J. 6:3891-3899.

van der Salm, T.P.M., C.H. Hänisch ten Cate, and H.J.M. Dons. 1996. Prospects for applications of rol genes for crop improvement. Plant Mol. Biol. Rpt. 14:207-228.

Winefield, C., D. Lewis, S. Arathoon, and S. Deroles. 1999. Alteration of Petunia plant form through the introduction of the rolC gene from Agrobacterium rhizogenes. Mol. Breeding 5:543-551.

Yokoyama, R., T. Hirose, N. Fujii, E.T. Aspuria, A. Kato, and H. Uchimiya. 1994. The rolC promoter of Agrobacterium rhizogenes Ri plasmid is activated by sucrose in transgenic tobacco plants. Mol. Gen. Genet. 244:15-22.

Zuker, A., T. Tzfira, G. Scovel, M. Ovadis, E. Shklarman, H. Itzhaki, and A. Vainstein. 2001. RolC-transgenic carnation with improved horticultural traits: Quantitative and qualitative analyses of greenhouse-grown plants. J. Amer. Soc. Hort. Sci. 126:13-18. 